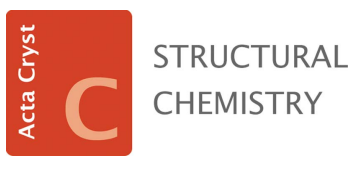

ISSN 2053-2296

Received 31 July 2020

Accepted 24 August 2020

Edited by M. Yousufuddin, University of North Texas at Dallas, USA

‡ Died 6th December 2019

Keywords: phenol; crystal structure; polymorph; thermochromism.

CCDC references: 2025079; 2025078

Supporting information: this article has supporting information at journals.iucr.org/C
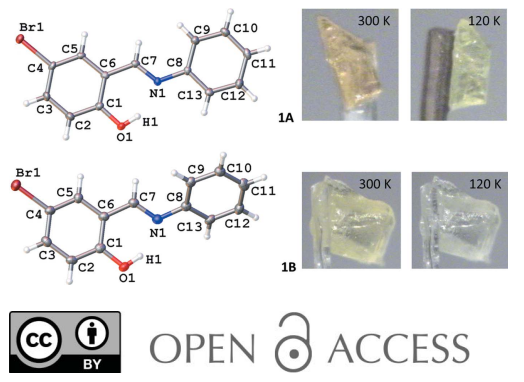

\section{(E)-4-Bromo-2-[(phenylimino)methyl]phenol: a new polymorph and thermochromism}

\author{
Helen E. Mason, ${ }^{a} \neq$ Judith A. K. Howard ${ }^{a}$ and Hazel A. Sparkes ${ }^{b *}$ \\ ${ }^{\mathbf{a}}$ Department of Chemistry, Durham University, South Road, Durham DH1 3LE, England, and ${ }^{\mathbf{b}}$ School of Chemistry, \\ University of Bristol, Cantock's Close, Bristol BS8 1TS, England. *Correspondence e-mail: hazel.sparkes@bristol.ac.uk
}

A new polymorph of (E)-4-bromo-2-[(phenylimino)methyl]phenol, $\mathrm{C}_{13} \mathrm{H}_{10} \mathrm{Br}$ $\mathrm{NO}$, is reported, together with a low-temperature structure determination of the previously published polymorph. Both polymorphs were found to have an intramolecular $\mathrm{O}-\mathrm{H} \cdots \mathrm{N}$ hydrogen bond between the phenol $\mathrm{OH}$ group and the imine $\mathrm{N}$ atom, forming an $S(6)$ ring. The crystals were observed to have different colours at room temperature, with the previously published polymorph being more orange and the new polymorph more yellow. The planarity of the molecule in the two polymorphs was found to be significantly different, with dihedral angles $(\Phi)$ between the two aromatic rings for the previously published 'orange' polymorph of $\Phi=1.8(2)^{\circ}$ at $120 \mathrm{~K}$, while the new 'yellow' polymorph had $\Phi=45.6(1)^{\circ}$ at $150 \mathrm{~K}$. It was also observed that both polymorphs displayed some degree of thermochromism and upon cooling the 'orange' polymorph became more yellow, while the 'yellow' polymorph became paler upon cooling.

\section{Introduction}

A wide range of $\mathrm{N}$-salicylideneanilines, Schiff bases of salicylaldehyde derivatives with aniline derivatives, have been synthesized (Özek et al., 2007; Johmoto et al., 2012). The $\mathrm{N}$-salicylideneaniline derivatives are interesting as they have generally been found to display thermochromism, with some also showing photochromism in the solid state (Cohen \& Schmidt, 1962; Cohen et al., 1964; Fujiwara et al., 2004). The mechanism for the chromic colour change is believed to be due to a keto-enol tautomerism (Hadjoudis \& Mavridis, 2004; Robert et al., 2009). The keto form is coloured, while the enol form is colourless and the switch can be induced either by changes in temperature or by irradiation. A link has been proposed between the thermochromic behaviour of a compound and the dihedral angle $(\Phi)$ between the two aromatic rings, with those having $\Phi<25^{\circ}$ being more likely to be strongly thermochromic (Hadjoudis \& Mavridis, 2004; Robert et al., 2009). A larger interplanar angle allows increased orbital overlap and greater delocalization into the $\pi$-system, which reduces the basicity of the $\mathrm{N}$ atom and thus the thermochromism. The effect of substituents on the $\mathrm{OH}$ bond strength, nitrogen-accepting ability and crystal packing have also been postulated as important in the chromic behaviour of the $N$-salicylideneanilines (Hadjoudis \& Mavridis, 2004; Robert $e t$ al., 2009). It has also been observed that, in general, the $\mathrm{N}$-salicylideneanilines that are more strongly coloured, typically red/orange, at room temperature, tend to be more strongly thermochromic than those that are paler, typically yellow, at room temperature (Ogawa et al., 2001; Fujiwara $e t$ al., 2009).

The structures of $(E)$-4-halogeno-2-[(phenylimino)methyl]phenol have been reported for fluoro (Swetha et al., 2017), 
Table 1

Experimental details.

For both structures: $\mathrm{C}_{13} \mathrm{H}_{10} \mathrm{BrNO}, M_{\mathrm{r}}=276.13, Z=4$. Experiments were carried out with Mo $K \alpha$ radiation using an Oxford Diffraction Xcalibur (Sapphire3, Gemini ultra) diffractometer. An analytical absorption correction [CrysAlis PRO (Oxford Diffraction, 2010), based on expressions derived by Clark \& Reid (1995)] was used. Refinement was with 2 restraints. H atoms were treated by a mixture of independent and constrained refinement.

\begin{tabular}{|c|c|c|}
\hline & Polymorph 1A & Polymorph 1B \\
\hline \multicolumn{3}{|l|}{ Crystal data } \\
\hline Crystal system, space group & Orthorhombic, $\mathrm{Pca}_{1}$ & Monoclinic, $C c$ \\
\hline Temperature $(\mathrm{K})$ & 120 & 150 \\
\hline$a, b, c(\AA)$ & $12.2768(3), 4.4829(1), 19.6694(4)$ & $25.8944(13), 6.9439(4), 6.1499(4)$ \\
\hline$\alpha, \beta, \gamma\left({ }^{\circ}\right)$ & $90,90,90$ & $90,91.381(5), 90$ \\
\hline$V\left(\AA^{3}\right)$ & $1082.52(4)$ & $1105.48(11)$ \\
\hline$\mu\left(\mathrm{mm}^{-1}\right)$ & 3.77 & 3.69 \\
\hline Crystal size $(\mathrm{mm})$ & $0.46 \times 0.20 \times 0.05$ & $0.58 \times 0.49 \times 0.22$ \\
\hline \multicolumn{3}{|l|}{ Data collection } \\
\hline$T_{\min }, T_{\max }$ & $0.383,0.847$ & $0.190,0.585$ \\
\hline $\begin{array}{l}\text { No. of measured, independent and observed } \\
\quad[I>2 \sigma(I)] \text { reflections }\end{array}$ & $13133,2215,2133$ & $7049,2254,2142$ \\
\hline$R_{\text {int }}$ & 0.043 & 0.051 \\
\hline$(\sin \theta / \lambda)_{\max }\left(\AA^{-1}\right)$ & 0.625 & 0.625 \\
\hline \multicolumn{3}{|l|}{ Refinement } \\
\hline$R\left[F^{2}>2 \sigma\left(F^{2}\right)\right], w R\left(F^{2}\right), S$ & $0.022,0.053,1.05$ & $0.039,0.100,1.05$ \\
\hline No. of reflections & 2215 & 2254 \\
\hline No. of parameters & 149 & 148 \\
\hline$\Delta \rho_{\max }, \Delta \rho_{\min }\left(\mathrm{e} \AA^{-3}\right)$ & $0.39,-0.23$ & $0.95,-0.34$ \\
\hline Absolute structure & $\begin{array}{l}\text { Flack } x \text { determined using } 993 \text { quotients } \\
\qquad\left[\left(I^{+}\right)-\left(I^{-}\right)\right] /\left[\left(I^{+}\right)+\left(I^{-}\right)\right] \text {(Parsons } \text { et al., 2013) }\end{array}$ & $\begin{array}{l}\text { Flack } x \text { determined using } 1007 \text { quotients } \\
\qquad\left[\left(I^{+}\right)-\left(I^{-}\right)\right] /\left[\left(I^{+}\right)+\left(I^{-}\right)\right] \text {(Parsons } \text { et al., 2013) }\end{array}$ \\
\hline Absolute structure parameter & $-0.006(8)$ & $-0.010(19)$ \\
\hline
\end{tabular}

Computer programs: CrysAlis PRO (Oxford Diffraction, 2010), SHELXS97 (Sheldrick, 2008), SHELXL2018 (Sheldrick, 2015) and OLEX (Dolomanov et al., 2009).

chloro (Bregman et al., 1964; Ogawa et al., 1998), bromo (Yan et al., 2014) and iodo (Swetha et al., 2019). Herein a new polymorph of (E)-4-bromo-2-[(phenylimino)methyl]phenol, denoted $\mathbf{1 B}$, is reported together with a new low-temperature determination of the previously reported polymorph, $\mathbf{1 A}$ (Yan et al., 2014). Both polymorphs were found to be thermochromic to some extent.

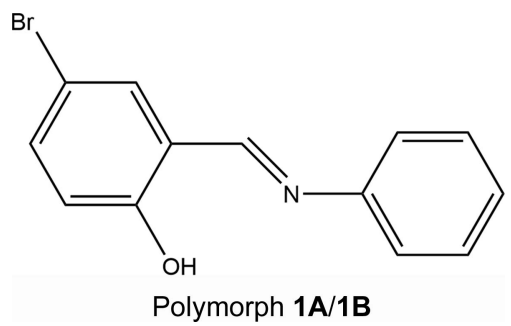

\section{Experimental}

\subsection{Synthesis and crystallization}

(E)-4-Bromo-2-[(phenylimino)methyl]phenol was synthesized by direct condensation of 5-bromosalicylaldehyde and aniline in ethanol. The two materials $(0.005 \mathrm{~mol}$ of each, $1.000 \mathrm{~g}$ of 5-bromosalicylaldehyde and $0.466 \mathrm{~g}$ of aniline) were dissolved separately in ethanol $(25 \mathrm{ml})$. The resultant solutions were combined and refluxed with stirring for $4 \mathrm{~h}$. After removal of any precipitate, the solution was rotary evaporated until further precipitate formed, the solid filtered off, rinsed with ethanol and left to dry, giving a yield of 94\% (1.304 g, $0.0047 \mathrm{~mol}$ ). Yellow single crystals (of 1B) crashed out of the crude reaction mixture and orange single crystals (of $\mathbf{1 A}$ ) were produced by recrystallization from ethanol.

\subsection{Refinement}

Crystal data, data collection and structure refinement details are summarized in Table 1 . All $\mathrm{H}$ atoms, apart from the $\mathrm{OH}$ hydrogen involved in the intramolecular hydrogen bonding with the imine $\mathrm{N}$ atom, were positioned geometrically and refined using a riding model. The $\mathrm{H}$ atoms involved in the intramolecular hydrogen bond were located in the Fourier difference map wherever feasible. In $\mathbf{1 A}$, the $\mathrm{O}-\mathrm{H}$ distance was restrained to 0.86 (1) .

\section{Results and discussion}

The structures of polymorphs $\mathbf{1 A}$ and $\mathbf{1 B}$ are shown in Fig. 1. The structure of $\mathbf{1 A}$ at $120 \mathrm{~K}$ was consistent with the previously published structure at room temperature (Yan et al., 2014). The structure of $\mathbf{1 A}$ was obtained in the orthorhombic space group $P c a 2_{1}$, while $\mathbf{1 B}$ was obtained in the monoclinic space group $C c$. The compound consists of a hydroxy-substituted phenyl ring linked via an imine group to a second unsubstituted phenyl group. In both polymorphs, the structures were found to exist in the enol form, with $\mathrm{C} 7=\mathrm{N} 1$ bond lengths of 1.282 (4) $\AA$ for $\mathbf{1 A}$ and 1.284 (10) $\AA$ for $\mathbf{1 B}$, indicating a double bond, and $\mathrm{C} 1-\mathrm{O} 1$ bond lengths of 1.350 (5) $\AA$ for $\mathbf{1 A}$ and 1.351 (9) $\AA$ for $\mathbf{1 B}$, indicating a single bond. The structures showed quite different dihedral angles, with $\mathbf{1 A}$ having $\Phi=1.8(2)^{\circ}$ at $120 \mathrm{~K}$ and $\mathbf{1 B}$ having $\Phi=$ 
Table 2

Hydrogen-bond geometry $\left(\AA,^{\circ}\right)$ for polymorph $\mathbf{1 A}$.

\begin{tabular}{lllll}
\hline$D-\mathrm{H} \cdots A$ & $D-\mathrm{H}$ & $\mathrm{H} \cdots A$ & $D \cdots A$ & $D-\mathrm{H} \cdots A$ \\
\hline $\mathrm{O} 1-\mathrm{H} 1 \cdots \mathrm{N} 1$ & $0.86(1)$ & $1.82(3)$ & $2.593(4)$ & $150(5)$ \\
\hline
\end{tabular}

Table 3

Hydrogen-bond geometry $\left(\AA{ }^{\circ}\right)$ for polymorph $\mathbf{1 B}$.

\begin{tabular}{lllll}
\hline$D-\mathrm{H} \cdots A$ & $D-\mathrm{H}$ & $\mathrm{H} \cdots A$ & $D \cdots A$ & $D-\mathrm{H} \cdots A$ \\
\hline $\mathrm{O} 1-\mathrm{H} 1 \cdots \mathrm{N} 1$ & $0.86(11)$ & $1.82(11)$ & $2.590(10)$ & $148(10)$ \\
\hline
\end{tabular}

$45.6(1)^{\circ}$ at $150 \mathrm{~K}$. Upon cooling, the structures were both found to display some degree of thermochromism with $\mathbf{1 A}$ changing from orange at room temperature to yellow at $120 \mathrm{~K}$ and 1B, which was yellow at room temperature, becoming slightly paler at $150 \mathrm{~K}$ (Fig. 2). The differences in the thermochromic behaviour of the two polymorphs are consistent with literature suggestions that a larger dihedral angle increases the overlap of the $\pi$-system reducing the nitrogen basicity, disfavouring the keto form and thus also reducing the thermochromism of the compound.

An intramolecular $\mathrm{O} 1-\mathrm{H} 1 \cdots \mathrm{N} 1$ hydrogen bond, involving the phenol $\mathrm{OH}$ group and imine $\mathrm{N}$ atom, was identified in the structures of both polymorphs and creates an $S(6)$ ring. The hydrogen-bonding parameters were almost identical in the two structures, with a donor-acceptor distance of $\sim 2.59 \AA$ and a hydrogen-bond angle of $\sim 150^{\circ}$ (Tables 2 and 3.). The packing of the two polymorphs was unsurprisingly significantly different given the large difference in the dihedral angles. In polymorph $\mathbf{1 A}$, the molecules are essentially planar

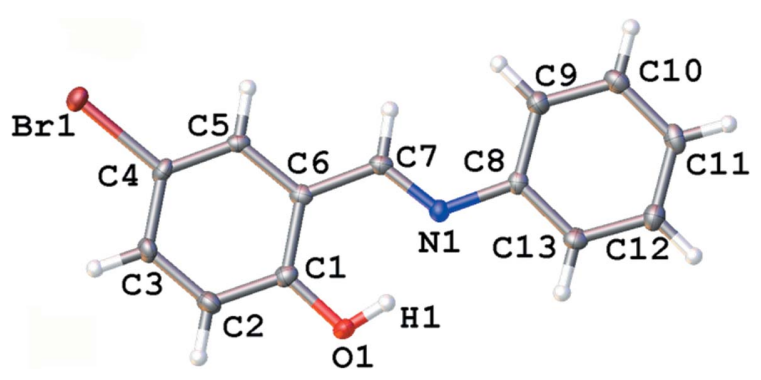

(a)

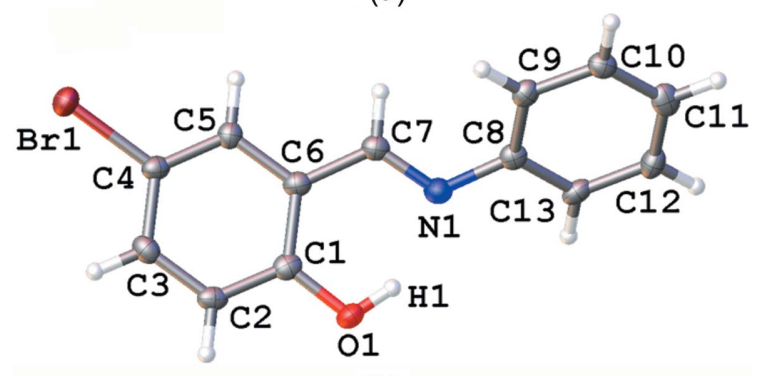

(b)

Figure 1

Illustration of the structures of $(a) \mathbf{1 A}$ and $(b) \mathbf{1 B}$ at $120(2)$ and 150 (2) K, respectively, with the atomic numbering schemes depicted. Anisotropic displacement parameters are shown at the $50 \%$ probability level.
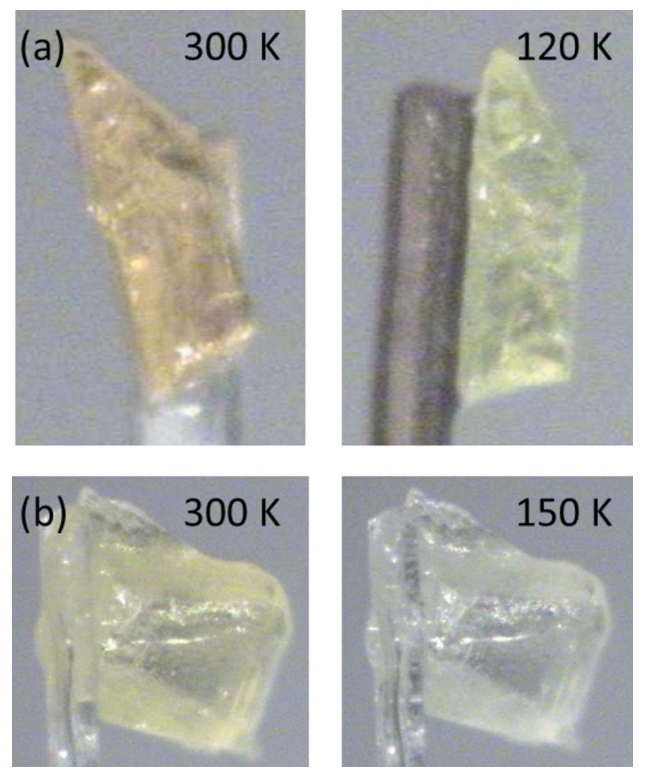

Figure 2

Illustration of the colour change observed upon cooling (a) $\mathbf{1 A}$ and $(b)$ $1 B$.

and orientated diagonally such that the plane of the molecule is perpendicular to the $b c$ plane and, as a result of the $2_{1}$ screw axis, the diagonal slant of alternate molecules along the $a$-axis direction essentially align in opposite directions (Fig. 3a). It was also noted that there were short $\pi$-type contacts between the $\mathrm{C}=\mathrm{N}$ group and the phenol ring in the $0 \overline{1} 1$ direction, with a centroid-to-centroid $(\mathrm{C}=\mathrm{N})$ distance of 3.326 (1) $\AA$. These can be seen on the Hirshfeld surface of $\mathbf{1 A}$ as red dots (Fig. 4a). In polymorph 1B, although the molecules themselves are
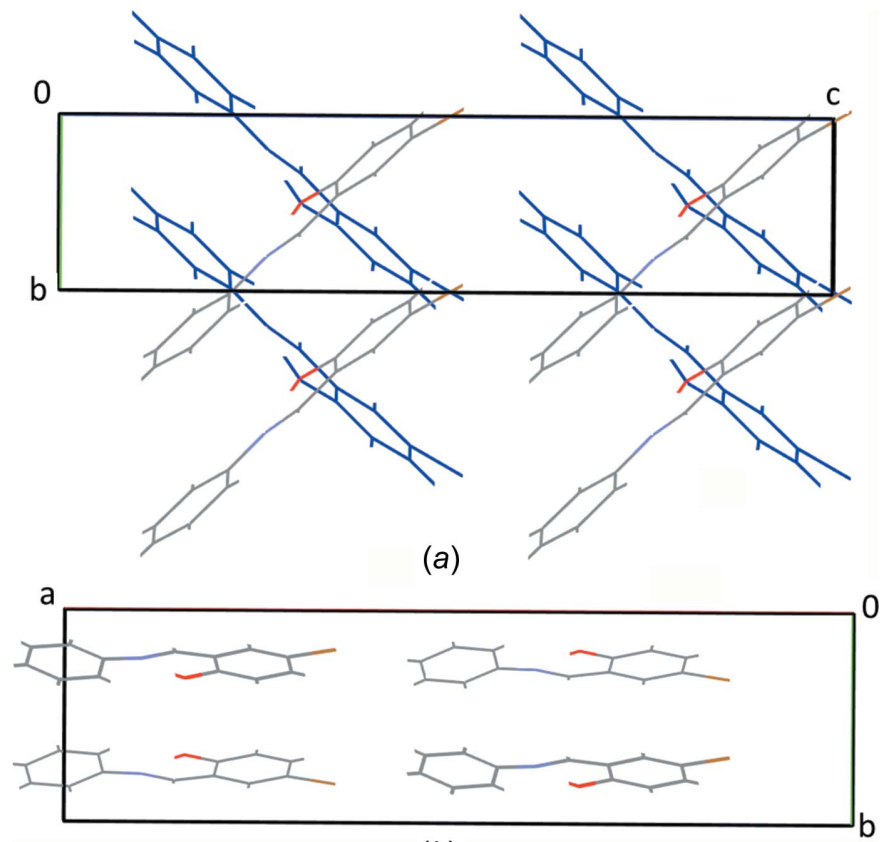

Figure 3

(b)

(a) Illustration of the packing for $\mathbf{1 A}$, looking down the $a$ axis; molecules in blue are in-plane behind those in element colours. (b) View of polymorph 1B, looking down the $c$ axis. 
twisted, the molecules are orientated relative to each other such that they create planes parallel to the $a c$ plane direction (see Fig. 3b).

Examining the Hirshfeld fingerprint plots (Turner et al., 2017) for the two structures highlights the differences in the two structures, not least in the shapes of the two plots (Fig. 4). For $\mathbf{1 A}$, the $\mathrm{O} \cdots \mathrm{H}$ and $\mathrm{Br} \cdots \mathrm{H}$ contacts are quite obvious, while in $\mathbf{1 B}$ the $\mathrm{H} \cdots \mathrm{H}$ and $\mathrm{C} \cdots \mathrm{H}$ contacts are significantly more pronounced, slightly masking the $\mathrm{O} \cdots \mathrm{H}$ and $\mathrm{Br} \cdots \mathrm{H}$ contacts. These differences are very apparent on the Hirshfeld surface for both compounds with a greater number of red spots on the surface of $\mathbf{1 A}$ that are more noticeable than for 1B, showing that $\mathbf{1 A}$ has more short contacts.

The two polymorphs of (E)-4-bromo-2-[(phenylimino)methyl]phenol reported herein are particularly interesting as part of a study into $N$-salicylideneanilines because they show significantly different molecular conformations and colours at room temperature. In line with the literature, the extent of the thermochromism was found to be linked to the dihedral angle, with $\mathbf{1 A}\left[\Phi=1.8(2)^{\circ}\right]$ showing a greater colour change upon cooling than observed for $\mathbf{1 B}\left[\Phi=45.6(1)^{\circ}\right]$.

\section{Acknowledgements}

The authors gratefully acknowledge funding for HEM from the EPSRC and from Durham University, and useful discussions with Professor Jonathan Steed of Durham University.

\section{References}

Bregman, J., Leiserowitz, L. \& Schmidt, G. M. J. (1964). J. Chem. Soc. pp. 2068-2085.

Clark, R. C. \& Reid, J. S. (1995). Acta Cryst. A51, 887-897.

Cohen, M. D. \& Schmidt, G. M. J. (1962). J. Phys. Chem. 66, 24422446.

Cohen, M. D., Schmidt, G. M. J. \& Flavian, S. (1964). J. Chem. Soc. pp. 2041-2051.

Dolomanov, O. V., Bourhis, L. J., Gildea, R. J., Howard, J. A. K. \& Puschmann, H. (2009). J. Appl. Cryst. 42, 339-341.

Fujiwara, T., Harada, J. \& Ogawa, K. (2004). J. Phys. Chem. B, 108, 4035-4038.

Fujiwara, T., Harada, J. \& Ogawa, K. (2009). J. Phys. Chem. A, 113, 1822-1826.

Hadjoudis, E. \& Mavridis, I. M. (2004). Chem. Soc. Rev. 33, 579-588.
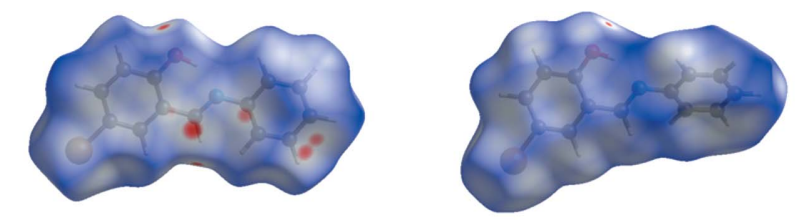

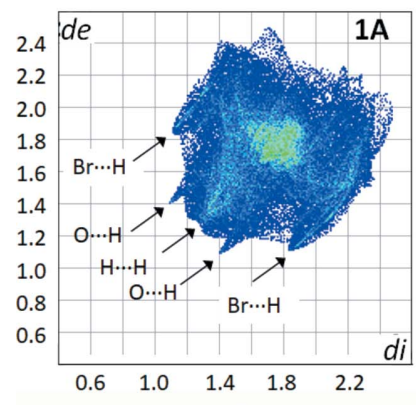

(a)

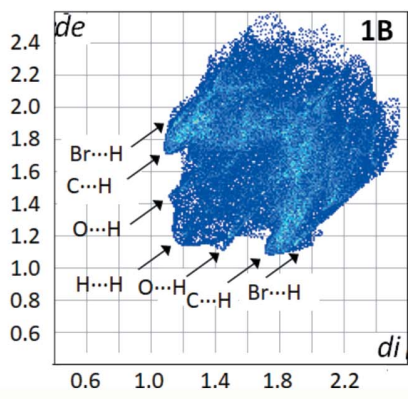

(b)
Figure 4

The Hirshfeld surface plot (top) and fingerprint plot (bottom) for (a) 1A and $(b) \mathbf{1 B}$.

Johmoto, K., Ishida, T., Sekine, A., Uekusa, H. \& Ohashi, Y. (2012). Acta Cryst. B68, 297-304.

Ogawa, K., Harada, J., Fujiwara, T. \& Yoshida, S. (2001). J. Phys. Chem. A, 105, 3425-3427.

Ogawa, K., Kasahara, Y., Ohtani, Y. \& Harada, J. (1998). J. Am. Chem. Soc. 120, 7107-7108.

Oxford Diffraction (2010). CrysAlis PRO. Oxford Diffraction Ltd, Abingdon, Oxfordshire, England.

Özek, A., Albayrak, C., Odabaşoğlu, M. \& Büyükgüngör, O. (2007). Acta Cryst. C63, o177-o180.

Parsons, S., Flack, H. D. \& Wagner, T. (2013). Acta Cryst. B69, 249 259.

Robert, F., Naik, A. D., Tinant, B., Robiette, R. \& Garcia, Y. (2009). Chem. Eur. J. 15, 4327-4342.

Sheldrick, G. M. (2008). Acta Cryst. A64, 112-122.

Sheldrick, G. M. (2015). Acta Cryst. C71, 3-8.

Swetha, G., Ida Malarselvi, R., Ramachandra Raja, C., Thiruvalluvar, A. \& Priscilla, J. (2017). IUCrData, 2, x171671.

Swetha, G., Ida Malarselvi, R., Ramachandra Raja, C., Thiruvalluvar, A. \& Priscilla, J. (2019). IUCrData, 4, x190788.

Turner, M. J., McKinnon, J. J., Wolff, S. K., Grimwood, D. J., Spackman, P. R., Jayatilaka, D. \& Spackman, M. A. (2017). CrystalExplorer17. University of Western Australia. https://crystalexplorer.scb.uwa.edu.au/.

Yan, X.-X., Lu, L. \& Zhu, M. (2014). Acta Cryst. E70, o853. 


\section{supporting information}

Acta Cryst. (2020). C76, 1001-1004 [https://doi.org/10.1107/S2053229620011560]

\section{(E)-4-Bromo-2-[(phenylimino) methyl]phenol: a new polymorph and}

\section{thermochromism}

\section{Helen E. Mason, Judith A. K. Howard and Hazel A. Sparkes}

Computing details

For both structures, data collection: CrysAlis PRO (Oxford Diffraction, 2010); cell refinement: CrysAlis PRO (Oxford Diffraction, 2010); data reduction: CrysAlis PRO (Oxford Diffraction, 2010); program(s) used to solve structure:

SHELXS97 (Sheldrick, 2008); program(s) used to refine structure: SHELXL2018 (Sheldrick, 2015); molecular graphics:

OLEX (Dolomanov et al., 2009); software used to prepare material for publication: OLEX (Dolomanov et al., 2009).

(E)-4-Bromo-2-((phenylimino)methyl]phenol (1A)

\section{Crystal data}

$\mathrm{C}_{13} \mathrm{H}_{10} \mathrm{BrNO}$

$M_{r}=276.13$

Orthorhombic, $P c a 2_{1}$

$a=12.2768(3) \AA$

$b=4.4829(1) \AA$

$c=19.6694(4) \AA$

$V=1082.52(4) \AA^{3}$

$Z=4$

$F(000)=552$

\section{Data collection}

Xcalibur, Sapphire3, Gemini ultra diffractometer

Radiation source: Enhance (Mo) X-ray Source Graphite monochromator

Detector resolution: 16.1511 pixels $\mathrm{mm}^{-1}$

$\omega$ scans

Absorption correction: analytical

[CrysAlis PRO (Oxford Diffraction, 2010), based on expressions derived by Clark \& Reid (1995)]

\section{Refinement}

Refinement on $F^{2}$

Least-squares matrix: full

$R\left[F^{2}>2 \sigma\left(F^{2}\right)\right]=0.022$

$w R\left(F^{2}\right)=0.053$

$S=1.05$

2215 reflections

149 parameters

2 restraints
$D_{\mathrm{x}}=1.694 \mathrm{Mg} \mathrm{m}^{-3}$

Mo $K \alpha$ radiation, $\lambda=0.71073 \AA$

Cell parameters from 5409 reflections

$\theta=2.7-30.6^{\circ}$

$\mu=3.77 \mathrm{~mm}^{-1}$

$T=120 \mathrm{~K}$

Block, orange

$0.46 \times 0.20 \times 0.05 \mathrm{~mm}$

$T_{\min }=0.383, T_{\max }=0.847$

13133 measured reflections

2215 independent reflections

2133 reflections with $I>2 \sigma(I)$

$R_{\text {int }}=0.043$

$\theta_{\text {max }}=26.4^{\circ}, \theta_{\min }=3.3^{\circ}$

$h=-15 \rightarrow 15$

$k=-5 \rightarrow 5$

$l=-24 \rightarrow 24$

Primary atom site location: structure-invariant direct methods

Hydrogen site location: mixed

$\mathrm{H}$ atoms treated by a mixture of independent and constrained refinement

$w=1 /\left[\sigma^{2}\left(F_{\mathrm{o}}^{2}\right)+(0.0288 P)^{2}+0.2498 P\right]$ where $P=\left(F_{\mathrm{o}}^{2}+2 F_{\mathrm{c}}^{2}\right) / 3$

$(\Delta / \sigma)_{\max }=0.001$

$\Delta \rho_{\max }=0.39 \mathrm{e} \AA^{-3}$ 
$\Delta \rho_{\min }=-0.23$ e $\AA^{-3}$

Special details

Geometry. All esds (except the esd in the dihedral angle between two 1.s. planes) are estimated using the full covariance matrix. The cell esds are taken into account individually in the estimation of esds in distances, angles and torsion angles; correlations between esds in cell parameters are only used when they are defined by crystal symmetry. An approximate (isotropic) treatment of cell esds is used for estimating esds involving l.s. planes.

Refinement. Single-crystal X-ray diffraction measurements for 1A were collected at 120 (2) K and 1B were collected at 150 (2) K on an Oxford Diffraction diffractometer. Both datasets were collected using Mo K $\alpha$ radiation $(\lambda=0.71073 \AA)$ and recorded on a CCD detector. The structures were solved using direct methods in ShelXS (Sheldrick, 2008). All structures were refined by full matrix least squares on $F^{2}$ using SHELXL (Sheldrick, 2008; Sheldrick, 2015) in Olex2 (Dolomanov et al., 2009).

Fractional atomic coordinates and isotropic or equivalent isotropic displacement parameters $\left(\AA^{2}\right)$

\begin{tabular}{|c|c|c|c|c|}
\hline & $x$ & $y$ & $z$ & $U_{\text {iso }} * / U_{\text {eq }}$ \\
\hline $\mathrm{Br} 1$ & $0.42487(2)$ & $1.04053(7)$ & $0.52257(3)$ & $0.01818(11)$ \\
\hline $\mathrm{C} 4$ & $0.3325(3)$ & $0.8715(8)$ & $0.45495(18)$ & $0.0151(7)$ \\
\hline $\mathrm{C} 5$ & $0.3746(3)$ & $0.6746(8)$ & $0.40824(17)$ & $0.0136(7)$ \\
\hline H5 & 0.449969 & 0.627095 & 0.409090 & $0.016^{*}$ \\
\hline $\mathrm{C} 3$ & $0.2233(3)$ & $0.9502(8)$ & $0.45376(19)$ & $0.0174(8)$ \\
\hline $\mathrm{H} 3$ & 0.195360 & 1.087904 & 0.486075 & $0.021 *$ \\
\hline C6 & $0.3066(3)$ & $0.5436(8)$ & $0.35936(19)$ & $0.0139(7)$ \\
\hline $\mathrm{C} 2$ & $0.1551(3)$ & $0.8270(9)$ & 0.40524 (19) & $0.0188(8)$ \\
\hline $\mathrm{H} 2$ & 0.080442 & 0.882022 & 0.403983 & $0.023^{*}$ \\
\hline $\mathrm{C} 1$ & $0.1956(3)$ & $0.6224(9)$ & $0.35818(18)$ & $0.0161(8)$ \\
\hline $\mathrm{O} 1$ & $0.1258(3)$ & $0.5046(6)$ & $0.31239(14)$ & $0.0199(6)$ \\
\hline $\mathrm{C} 7$ & $0.3514(3)$ & $0.3292(8)$ & $0.31122(18)$ & $0.0145(7)$ \\
\hline $\mathrm{H} 7$ & 0.427300 & 0.288024 & 0.311612 & $0.017 *$ \\
\hline N1 & $0.2898(2)$ & $0.1953(6)$ & $0.26834(14)$ & $0.0138(6)$ \\
\hline C8 & $0.3316(3)$ & $-0.0169(7)$ & $0.2211(2)$ & $0.0139(8)$ \\
\hline $\mathrm{C} 13$ & $0.2578(3)$ & $-0.1317(8)$ & $0.17480(18)$ & $0.0171(7)$ \\
\hline H13 & 0.184046 & -0.068148 & 0.175947 & $0.021 *$ \\
\hline $\mathrm{C} 11$ & $0.3978(4)$ & $-0.4362(8)$ & $0.1251(2)$ & $0.0193(8)$ \\
\hline H11 & 0.420342 & -0.577964 & 0.092026 & $0.023 *$ \\
\hline C9 & $0.4398(3)$ & $-0.1160(9)$ & $0.2201(2)$ & $0.0176(8)$ \\
\hline H9 & 0.490778 & -0.040319 & 0.252150 & $0.021 *$ \\
\hline $\mathrm{C} 10$ & $0.4724(3)$ & $-0.3257(8)$ & 0.17207 (19) & $0.0187(8)$ \\
\hline $\mathrm{H} 10$ & 0.545717 & -0.393545 & 0.171327 & $0.022 *$ \\
\hline $\mathrm{C} 12$ & $0.2912(3)$ & $-0.3398(9)$ & $0.12650(19)$ & $0.0200(8)$ \\
\hline H12 & 0.240293 & -0.415470 & 0.094460 & $0.024^{*}$ \\
\hline H1 & $0.161(3)$ & $0.369(8)$ & $0.291(2)$ & $0.036(14)^{*}$ \\
\hline
\end{tabular}

Atomic displacement parameters $\left(\AA^{2}\right)$

\begin{tabular}{lllllll}
\hline & $U^{11}$ & $U^{22}$ & $U^{33}$ & $U^{12}$ & $U^{13}$ & $U^{23}$ \\
\hline $\operatorname{Br} 1$ & $0.01826(17)$ & $0.02317(18)$ & $0.01312(16)$ & $-0.00238(12)$ & $-0.0012(2)$ & $-0.0025(3)$
\end{tabular}


supporting information

\begin{tabular}{lllllll} 
C4 & $0.0181(19)$ & $0.0169(17)$ & $0.0104(17)$ & $-0.0043(15)$ & $-0.0021(13)$ & $0.0006(15)$ \\
C5 & $0.0115(17)$ & $0.0143(17)$ & $0.0149(17)$ & $-0.0001(14)$ & $-0.0009(14)$ & $0.0041(14)$ \\
C3 & $0.019(2)$ & $0.0194(18)$ & $0.0138(18)$ & $0.0009(15)$ & $0.0035(16)$ & $-0.0029(15)$ \\
C6 & $0.0147(18)$ & $0.0149(17)$ & $0.0122(18)$ & $0.0005(14)$ & $0.0009(14)$ & $0.0048(14)$ \\
C2 & $0.0167(19)$ & $0.0221(19)$ & $0.0175(19)$ & $0.0039(16)$ & $0.0023(15)$ & $0.0003(16)$ \\
C1 & $0.0161(18)$ & $0.0187(18)$ & $0.0135(18)$ & $-0.0012(15)$ & $-0.0011(15)$ & $0.0029(15)$ \\
O1 & $0.0129(14)$ & $0.0282(15)$ & $0.0186(14)$ & $0.0013(11)$ & $-0.0026(11)$ & $-0.0074(12)$ \\
C7 & $0.0129(17)$ & $0.0149(17)$ & $0.0156(17)$ & $0.0004(15)$ & $0.0007(14)$ & $0.0036(15)$ \\
N1 & $0.0157(15)$ & $0.0138(15)$ & $0.0120(13)$ & $0.0003(11)$ & $0.0005(13)$ & $0.0007(12)$ \\
C8 & $0.017(2)$ & $0.0127(17)$ & $0.0115(18)$ & $-0.0014(14)$ & $0.0011(13)$ & $0.0018(14)$ \\
C13 & $0.0175(19)$ & $0.0157(16)$ & $0.0181(18)$ & $0.0025(15)$ & $-0.0012(15)$ & $0.0031(15)$ \\
C11 & $0.028(2)$ & $0.0154(19)$ & $0.0144(19)$ & $0.0009(16)$ & $0.0066(16)$ & $0.0014(15)$ \\
C9 & $0.0182(19)$ & $0.0170(18)$ & $0.0176(19)$ & $0.0005(15)$ & $0.0007(14)$ & $-0.0001(15)$ \\
C10 & $0.0173(19)$ & $0.0197(19)$ & $0.0190(19)$ & $0.0019(15)$ & $0.0048(15)$ & $0.0026(16)$ \\
C12 & $0.025(2)$ & $0.0184(19)$ & $0.0170(18)$ & $-0.0016(16)$ & $-0.0010(16)$ & $-0.0016(16)$ \\
& & & & & & \\
\hline
\end{tabular}

Geometric parameters $\left(A,{ }^{\circ}\right)$

\begin{tabular}{|c|c|c|c|}
\hline $\mathrm{Br} 1-\mathrm{C} 4$ & $1.905(4)$ & $\mathrm{C} 7-\mathrm{N} 1$ & $1.282(4)$ \\
\hline $\mathrm{C} 4-\mathrm{C} 5$ & $1.375(5)$ & $\mathrm{N} 1-\mathrm{C} 8$ & $1.425(5)$ \\
\hline $\mathrm{C} 4-\mathrm{C} 3$ & $1.387(5)$ & $\mathrm{C} 8-\mathrm{C} 13$ & $1.384(5)$ \\
\hline $\mathrm{C} 5-\mathrm{H} 5$ & 0.9500 & $\mathrm{C} 8-\mathrm{C} 9$ & $1.401(5)$ \\
\hline $\mathrm{C} 5-\mathrm{C} 6$ & $1.402(5)$ & $\mathrm{C} 13-\mathrm{H} 13$ & 0.9500 \\
\hline $\mathrm{C} 3-\mathrm{H} 3$ & 0.9500 & $\mathrm{C} 13-\mathrm{C} 12$ & $1.393(5)$ \\
\hline $\mathrm{C} 3-\mathrm{C} 2$ & $1.384(6)$ & $\mathrm{C} 11-\mathrm{H} 11$ & 0.9500 \\
\hline $\mathrm{C} 6-\mathrm{C} 1$ & $1.408(5)$ & $\mathrm{C} 11-\mathrm{C} 10$ & $1.393(6)$ \\
\hline $\mathrm{C} 6-\mathrm{C} 7$ & $1.457(5)$ & $\mathrm{C} 11-\mathrm{C} 12$ & $1.379(6)$ \\
\hline $\mathrm{C} 2-\mathrm{H} 2$ & 0.9500 & C9-H9 & 0.9500 \\
\hline $\mathrm{C} 2-\mathrm{C} 1$ & $1.394(5)$ & $\mathrm{C} 9-\mathrm{C} 10$ & $1.392(5)$ \\
\hline $\mathrm{C} 1-\mathrm{O} 1$ & $1.350(5)$ & $\mathrm{C} 10-\mathrm{H} 10$ & 0.9500 \\
\hline $\mathrm{O} 1-\mathrm{H} 1$ & $0.857(14)$ & $\mathrm{C} 12-\mathrm{H} 12$ & 0.9500 \\
\hline $\mathrm{C} 7-\mathrm{H} 7$ & 0.9500 & & \\
\hline $\mathrm{C} 5-\mathrm{C} 4-\mathrm{Br} 1$ & $119.9(3)$ & $\mathrm{N} 1-\mathrm{C} 7-\mathrm{H} 7$ & 119.5 \\
\hline $\mathrm{C} 5-\mathrm{C} 4-\mathrm{C} 3$ & $121.0(3)$ & $\mathrm{C} 7-\mathrm{N} 1-\mathrm{C} 8$ & $121.9(3)$ \\
\hline $\mathrm{C} 3-\mathrm{C} 4-\mathrm{Br} 1$ & $119.1(3)$ & $\mathrm{C} 13-\mathrm{C} 8-\mathrm{N} 1$ & $116.2(3)$ \\
\hline $\mathrm{C} 4-\mathrm{C} 5-\mathrm{H} 5$ & 119.9 & $\mathrm{C} 13-\mathrm{C} 8-\mathrm{C} 9$ & $119.6(4)$ \\
\hline $\mathrm{C} 4-\mathrm{C} 5-\mathrm{C} 6$ & $120.2(3)$ & $\mathrm{C} 9-\mathrm{C} 8-\mathrm{N} 1$ & $124.2(3)$ \\
\hline $\mathrm{C} 6-\mathrm{C} 5-\mathrm{H} 5$ & 119.9 & $\mathrm{C} 8-\mathrm{C} 13-\mathrm{H} 13$ & 119.8 \\
\hline $\mathrm{C} 4-\mathrm{C} 3-\mathrm{H} 3$ & 120.2 & $\mathrm{C} 8-\mathrm{C} 13-\mathrm{C} 12$ & $120.4(4)$ \\
\hline $\mathrm{C} 2-\mathrm{C} 3-\mathrm{C} 4$ & $119.6(3)$ & $\mathrm{C} 12-\mathrm{C} 13-\mathrm{H} 13$ & 119.8 \\
\hline $\mathrm{C} 2-\mathrm{C} 3-\mathrm{H} 3$ & 120.2 & $\mathrm{C} 10-\mathrm{C} 11-\mathrm{H} 11$ & 120.0 \\
\hline $\mathrm{C} 5-\mathrm{C} 6-\mathrm{C} 1$ & $118.8(3)$ & $\mathrm{C} 12-\mathrm{C} 11-\mathrm{H} 11$ & 120.0 \\
\hline $\mathrm{C} 5-\mathrm{C} 6-\mathrm{C} 7$ & $119.8(3)$ & $\mathrm{C} 12-\mathrm{C} 11-\mathrm{C} 10$ & $119.9(4)$ \\
\hline $\mathrm{C} 1-\mathrm{C} 6-\mathrm{C} 7$ & $121.3(3)$ & $\mathrm{C} 8-\mathrm{C} 9-\mathrm{H} 9$ & 120.1 \\
\hline $\mathrm{C} 3-\mathrm{C} 2-\mathrm{H} 2$ & 119.8 & $\mathrm{C} 10-\mathrm{C} 9-\mathrm{C} 8$ & $119.7(4)$ \\
\hline $\mathrm{C} 3-\mathrm{C} 2-\mathrm{C} 1$ & $120.3(4)$ & $\mathrm{C} 10-\mathrm{C} 9-\mathrm{H} 9$ & 120.1 \\
\hline $\mathrm{C} 1-\mathrm{C} 2-\mathrm{H} 2$ & 119.8 & $\mathrm{C} 11-\mathrm{C} 10-\mathrm{H} 10$ & 119.9 \\
\hline
\end{tabular}




$\begin{array}{llll}\mathrm{C} 2-\mathrm{C} 1-\mathrm{C} 6 & 119.9(3) & \mathrm{C} 9-\mathrm{C} 10-\mathrm{C} 11 & 120.1(4) \\ \mathrm{O} 1-\mathrm{C} 1-\mathrm{C} 6 & 121.8(3) & \mathrm{C} 9-\mathrm{C} 10-\mathrm{H} 10 & 119.9 \\ \mathrm{O} 1-\mathrm{C} 1-\mathrm{C} 2 & 118.3(3) & \mathrm{C} 13-\mathrm{C} 12-\mathrm{H} 12 & 119.9 \\ \mathrm{C} 1-\mathrm{O} 1-\mathrm{H} 1 & 107(3) & \mathrm{C} 11-\mathrm{C} 12-\mathrm{C} 13 & 120.2(4) \\ \mathrm{C} 6-\mathrm{C} 7-\mathrm{H} 7 & \mathrm{C} 11-\mathrm{C} 12-\mathrm{H} 12 & \\ \mathrm{~N} 1-\mathrm{C} 7-\mathrm{C} 6 & 119.5 & & \\ & 120.9(3) & \mathrm{C} 1-\mathrm{C} 6-\mathrm{C} 7-\mathrm{N} 1 & -3.3(5) \\ \mathrm{Br} 1-\mathrm{C} 4-\mathrm{C} 5-\mathrm{C} 6 & 178.4(3) & \mathrm{C} 7-\mathrm{C} 6-\mathrm{C} 1-\mathrm{C} 2 & 179.6(3) \\ \mathrm{Br} 1-\mathrm{C} 4-\mathrm{C} 3-\mathrm{C} 2 & -179.5(3) & \mathrm{C} 7-\mathrm{C} 6-\mathrm{C} 1-\mathrm{O} 1 & -0.3(6) \\ \mathrm{C} 4-\mathrm{C} 5-\mathrm{C} 6-\mathrm{C} 1 & 1.4(5) & \mathrm{C} 7-\mathrm{N} 1-\mathrm{C} 8-\mathrm{C} 13 & 5.1(5) \\ \mathrm{C} 4-\mathrm{C} 5-\mathrm{C} 6-\mathrm{C} 7 & -178.4(3) & \mathrm{C} 7-\mathrm{N} 1-\mathrm{C} 8-\mathrm{C} 9 & 179.8(3) \\ \mathrm{C} 4-\mathrm{C} 3-\mathrm{C} 2-\mathrm{C} 1 & 0.7(6) & \mathrm{N} 1-\mathrm{C} 8-\mathrm{C} 13-\mathrm{C} 9-\mathrm{C} 12 & 179.6(3) \\ \mathrm{C} 5-\mathrm{C} 4-\mathrm{C} 3-\mathrm{C} 2 & 0.6(6) & \mathrm{C} 8-\mathrm{C} 13-\mathrm{C} 12-\mathrm{C} 11 & 0.9(6) \\ \mathrm{C} 5-\mathrm{C} 6-\mathrm{C} 1-\mathrm{C} 2 & -0.1(5) & \mathrm{C} 8-\mathrm{C} 9-\mathrm{C} 10-\mathrm{C} 11 & 0.1(6) \\ \mathrm{C} 5-\mathrm{C} 6-\mathrm{C} 1-\mathrm{O} 1 & 179.9(3) & \mathrm{C} 13-\mathrm{C} 8-\mathrm{C} 9-\mathrm{C} 10 & 0.8(6) \\ \mathrm{C} 5-\mathrm{C} 6-\mathrm{C} 7-\mathrm{N} 1 & 176.4(3) & \mathrm{C} 9-\mathrm{C} 8-\mathrm{C} 13-\mathrm{C} 12 & -1.3(6) \\ \mathrm{C} 3-\mathrm{C} 4-\mathrm{C} 5-\mathrm{C} 6 & -1.6(5) & \mathrm{C} 10-\mathrm{C} 11-\mathrm{C} 12-\mathrm{C} 13 & 0.1(5) \\ \mathrm{C} 3-\mathrm{C} 2-\mathrm{C} 1-\mathrm{C} 6 & -0.9(6) & \mathrm{C} 12-\mathrm{C} 11-\mathrm{C} 10-\mathrm{C} 9 & -0.6(5) \\ \mathrm{C} 3-\mathrm{C} 2-\mathrm{C} 1-\mathrm{O} 1 & 179.0(3) & & \\ \mathrm{C} 6-\mathrm{C} 7-\mathrm{N} 1-\mathrm{C} 8 & -179.5(3) & & \\ \end{array}$

Hydrogen-bond geometry $\left(A,{ }^{\circ}\right)$

\begin{tabular}{lllll}
\hline$D-\mathrm{H} \cdots A$ & $D-\mathrm{H}$ & $\mathrm{H} \cdots A$ & $D \cdots A$ & $D-\mathrm{H} \cdots A$ \\
\hline $\mathrm{O} 1-\mathrm{H} 1 \cdots \mathrm{N} 1$ & $0.86(1)$ & $1.82(3)$ & $2.593(4)$ & $150(5)$ \\
\hline
\end{tabular}

(E)-4-Bromo-2-((phenylimino)methyl]phenol (1B)

Crystal data

$\mathrm{C}_{13} \mathrm{H}_{10} \mathrm{BrNO}$

$M_{r}=276.13$

Monoclinic, $C c$

$a=25.8944$ (13) $\AA$

$b=6.9439(4) \AA$

$c=6.1499(4) \AA$

$\beta=91.381(5)^{\circ}$

$V=1105.48(11) \AA^{3}$

$Z=4$

\section{Data collection}

Xcalibur, Sapphire3, Gemini ultra diffractometer

Radiation source: Enhance (Mo) X-ray Source Graphite monochromator

Detector resolution: 16.1511 pixels $\mathrm{mm}^{-1}$

$\omega$ scans

Absorption correction: analytical

[CrysAlis PRO (Oxford Diffraction, 2010),

based on expressions derived by Clark \& Reid (1995)]
$F(000)=552$

$D_{\mathrm{x}}=1.659 \mathrm{Mg} \mathrm{m}^{-3}$

Mo $K \alpha$ radiation, $\lambda=0.71073 \AA$

Cell parameters from 3202 reflections

$\theta=3.0-30.7^{\circ}$

$\mu=3.69 \mathrm{~mm}^{-1}$

$T=150 \mathrm{~K}$

Block, yellow

$0.58 \times 0.49 \times 0.22 \mathrm{~mm}$

$T_{\min }=0.190, T_{\max }=0.585$

7049 measured reflections

2254 independent reflections

2142 reflections with $I>2 \sigma(I)$

$R_{\text {int }}=0.051$

$\theta_{\max }=26.4^{\circ}, \theta_{\min }=3.0^{\circ}$

$h=-32 \rightarrow 32$

$k=-8 \rightarrow 8$

$l=-7 \rightarrow 7$ 


\section{Refinement}

Refinement on $F^{2}$

Least-squares matrix: full

$R\left[F^{2}>2 \sigma\left(F^{2}\right)\right]=0.039$

$w R\left(F^{2}\right)=0.100$

$S=1.05$

2254 reflections

148 parameters

2 restraints

Primary atom site location: structure-invariant direct methods

Hydrogen site location: mixed
$\mathrm{H}$ atoms treated by a mixture of independent and constrained refinement

$w=1 /\left[\sigma^{2}\left(F_{\mathrm{o}}^{2}\right)+(0.067 P)^{2}\right]$

where $P=\left(F_{\mathrm{o}}^{2}+2 F_{\mathrm{c}}^{2}\right) / 3$

$(\Delta / \sigma)_{\max }<0.001$

$\Delta \rho_{\max }=0.95$ e $\AA^{-3}$

$\Delta \rho_{\min }=-0.34$ e $\AA^{-3}$

Absolute structure: Flack $x$ determined using 1007 quotients $[(\mathrm{I}+)-(\mathrm{I}-)] /[(\mathrm{I}+)+(\mathrm{I}-)]$ (Parsons et al., 2013)

Absolute structure parameter: $-0.010(19)$

Special details

Geometry. All esds (except the esd in the dihedral angle between two 1.s. planes) are estimated using the full covariance matrix. The cell esds are taken into account individually in the estimation of esds in distances, angles and torsion angles; correlations between esds in cell parameters are only used when they are defined by crystal symmetry. An approximate (isotropic) treatment of cell esds is used for estimating esds involving l.s. planes.

Fractional atomic coordinates and isotropic or equivalent isotropic displacement parameters $\left(\AA^{2}\right)$

\begin{tabular}{lllll}
\hline & $x$ & $y$ & $z$ & $U_{\text {iso }} / U_{\text {eq }}$ \\
\hline Br1 & $0.15729(2)$ & $0.68317(7)$ & $0.23237(2)$ & $0.0279(2)$ \\
C5 & $0.2655(3)$ & $0.6834(8)$ & $0.3219(12)$ & $0.0199(13)$ \\
H5 & 0.268030 & 0.635213 & 0.177996 & $0.024^{*}$ \\
C2 & $0.2577(3)$ & $0.8229(8)$ & $0.7462(12)$ & $0.0211(15)$ \\
H2 & 0.254820 & 0.870832 & 0.890033 & $0.025^{*}$ \\
C4 & $0.2176(3)$ & $0.7189(9)$ & $0.4073(11)$ & $0.0209(13)$ \\
C3 & $0.2131(3)$ & $0.7895(8)$ & $0.6189(12)$ & $0.0227(13)$ \\
H3 & 0.179986 & 0.814594 & 0.675762 & $0.027^{*}$ \\
C6 & $0.3105(3)$ & $0.7189(9)$ & $0.4487(11)$ & $0.0205(13)$ \\
O1 & $0.3481(2)$ & $0.8190(6)$ & $0.7919(9)$ & $0.0261(11)$ \\
H1 & $0.375(4)$ & $0.789(12)$ & $0.720(18)$ & $0.031^{*}$ \\
N1 & $0.4028(3)$ & $0.7276(11)$ & $0.4603(12)$ & $0.0217(14)$ \\
C7 & $0.3609(3)$ & $0.6969(8)$ & $0.3496(12)$ & $0.0206(13)$ \\
H7 & 0.362760 & 0.659495 & 0.201366 & $0.025^{*}$ \\
C1 & $0.3059(3)$ & $0.7863(8)$ & $0.6634(12)$ & $0.0214(13)$ \\
C8 & $0.4511(3)$ & $0.7333(9)$ & $0.3593(11)$ & $0.0201(12)$ \\
C10 & $0.5062(3)$ & $0.8319(8)$ & $0.0687(12)$ & $0.0225(14)$ \\
H10 & 0.510473 & 0.889386 & -0.069899 & $0.027^{*}$ \\
C9 & $0.4569(3)$ & $0.8171(8)$ & $0.1546(12)$ & $0.0217(14)$ \\
H9 & 0.427585 & 0.863641 & 0.074706 & $0.026^{*}$ \\
C12 & $0.5427(3)$ & $0.6791(9)$ & $0.3880(13)$ & $0.0248(15)$ \\
H12 & 0.571957 & 0.630545 & 0.466579 & $0.030^{*}$ \\
C13 & $0.4940(3)$ & $0.6661(8)$ & $0.4764(12)$ & $0.0221(14)$ \\
H13 & 0.490036 & 0.611519 & 0.616581 & $0.027^{*}$ \\
C11 & $0.5489(3)$ & $0.7631(11)$ & $0.1843(13)$ & $0.0257(15)$ \\
H11 & 0.582359 & 0.773163 & 0.124973 & $0.031^{*}$ \\
& & & &
\end{tabular}


Atomic displacement parameters $\left(\AA^{2}\right)$

\begin{tabular}{lllllll}
\hline & $U^{11}$ & $U^{22}$ & $U^{33}$ & $U^{12}$ & $U^{13}$ & $U^{23}$ \\
\hline Br1 & $0.0212(3)$ & $0.0346(3)$ & $0.0276(3)$ & $0.0000(4)$ & $-0.0036(2)$ & $-0.0023(4)$ \\
C5 & $0.020(3)$ & $0.017(3)$ & $0.023(4)$ & $0.000(2)$ & $0.000(3)$ & $0.002(2)$ \\
C2 & $0.030(4)$ & $0.016(3)$ & $0.017(4)$ & $-0.004(3)$ & $0.001(3)$ & $0.000(2)$ \\
C4 & $0.025(3)$ & $0.020(3)$ & $0.018(3)$ & $-0.003(2)$ & $0.000(2)$ & $0.000(2)$ \\
C3 & $0.025(3)$ & $0.020(3)$ & $0.024(3)$ & $0.004(2)$ & $0.005(3)$ & $0.002(2)$ \\
C6 & $0.025(3)$ & $0.017(3)$ & $0.020(3)$ & $0.001(2)$ & $-0.001(2)$ & $0.000(2)$ \\
O1 & $0.023(3)$ & $0.035(3)$ & $0.021(3)$ & $0.0002(19)$ & $-0.004(2)$ & $-0.0046(19)$ \\
N1 & $0.025(3)$ & $0.020(3)$ & $0.020(3)$ & $0.003(2)$ & $0.000(2)$ & $0.001(2)$ \\
C7 & $0.025(4)$ & $0.017(3)$ & $0.020(3)$ & $0.000(2)$ & $0.001(3)$ & $0.000(2)$ \\
C1 & $0.027(4)$ & $0.017(3)$ & $0.021(3)$ & $-0.001(2)$ & $-0.001(3)$ & $0.004(2)$ \\
C8 & $0.024(3)$ & $0.014(3)$ & $0.022(3)$ & $0.000(2)$ & $0.000(2)$ & $-0.001(2)$ \\
C10 & $0.027(4)$ & $0.019(3)$ & $0.022(3)$ & $-0.002(2)$ & $0.003(3)$ & $0.000(2)$ \\
C9 & $0.023(3)$ & $0.017(3)$ & $0.025(4)$ & $-0.001(2)$ & $-0.002(3)$ & $-0.001(2)$ \\
C12 & $0.020(4)$ & $0.023(3)$ & $0.031(4)$ & $0.001(2)$ & $-0.004(3)$ & $-0.001(2)$ \\
C13 & $0.026(4)$ & $0.018(3)$ & $0.021(4)$ & $-0.001(2)$ & $-0.007(3)$ & $-0.001(2)$ \\
C11 & $0.026(4)$ & $0.016(4)$ & $0.036(4)$ & $-0.005(3)$ & $0.004(3)$ & $-0.001(3)$ \\
& & & & & & \\
\hline
\end{tabular}

Geometric parameters $(\AA, \stackrel{\circ}{)})$

\begin{tabular}{|c|c|c|c|}
\hline $\mathrm{Br} 1-\mathrm{C} 4$ & $1.891(7)$ & $\mathrm{N} 1-\mathrm{C} 8$ & $1.410(10)$ \\
\hline $\mathrm{C} 5-\mathrm{H} 5$ & 0.9500 & $\mathrm{C} 7-\mathrm{H} 7$ & 0.9500 \\
\hline $\mathrm{C} 5-\mathrm{C} 4$ & $1.380(10)$ & $\mathrm{C} 8-\mathrm{C} 9$ & $1.398(10)$ \\
\hline $\mathrm{C} 5-\mathrm{C} 6$ & $1.409(10)$ & $\mathrm{C} 8-\mathrm{C} 13$ & $1.391(10)$ \\
\hline $\mathrm{C} 2-\mathrm{H} 2$ & 0.9500 & $\mathrm{C} 10-\mathrm{H} 10$ & 0.9500 \\
\hline $\mathrm{C} 2-\mathrm{C} 3$ & $1.398(11)$ & $\mathrm{C} 10-\mathrm{C} 9$ & $1.398(10)$ \\
\hline $\mathrm{C} 2-\mathrm{C} 1$ & $1.383(11)$ & $\mathrm{C} 10-\mathrm{C} 11$ & $1.385(11)$ \\
\hline $\mathrm{C} 4-\mathrm{C} 3$ & $1.398(9)$ & C9- $\mathrm{H} 9$ & 0.9500 \\
\hline $\mathrm{C} 3-\mathrm{H} 3$ & 0.9500 & $\mathrm{C} 12-\mathrm{H} 12$ & 0.9500 \\
\hline $\mathrm{C} 6-\mathrm{C} 7$ & $1.463(9)$ & $\mathrm{C} 12-\mathrm{C} 13$ & $1.388(11)$ \\
\hline $\mathrm{C} 6-\mathrm{C} 1$ & $1.409(10)$ & $\mathrm{C} 12-\mathrm{C} 11$ & $1.395(11)$ \\
\hline $\mathrm{O} 1-\mathrm{H} 1$ & $0.86(11)$ & $\mathrm{C} 13-\mathrm{H} 13$ & 0.9500 \\
\hline $\mathrm{O} 1-\mathrm{C} 1$ & $1.351(9)$ & $\mathrm{C} 11-\mathrm{H} 11$ & 0.9500 \\
\hline $\mathrm{N} 1-\mathrm{C} 7$ & $1.284(10)$ & & \\
\hline $\mathrm{C} 4-\mathrm{C} 5-\mathrm{H} 5$ & 120.1 & $\mathrm{O} 1-\mathrm{C} 1-\mathrm{C} 2$ & $118.5(7)$ \\
\hline $\mathrm{C} 4-\mathrm{C} 5-\mathrm{C} 6$ & $119.8(6)$ & $\mathrm{O} 1-\mathrm{C} 1-\mathrm{C} 6$ & $121.2(6)$ \\
\hline $\mathrm{C} 6-\mathrm{C} 5-\mathrm{H} 5$ & 120.1 & $\mathrm{C} 9-\mathrm{C} 8-\mathrm{N} 1$ & $121.6(7)$ \\
\hline $\mathrm{C} 3-\mathrm{C} 2-\mathrm{H} 2$ & 119.9 & $\mathrm{C} 13-\mathrm{C} 8-\mathrm{N} 1$ & $118.0(7)$ \\
\hline $\mathrm{C} 1-\mathrm{C} 2-\mathrm{H} 2$ & 119.9 & $\mathrm{C} 13-\mathrm{C} 8-\mathrm{C} 9$ & $120.2(6)$ \\
\hline $\mathrm{C} 1-\mathrm{C} 2-\mathrm{C} 3$ & $120.3(7)$ & $\mathrm{C} 9-\mathrm{C} 10-\mathrm{H} 10$ & 119.8 \\
\hline $\mathrm{C} 5-\mathrm{C} 4-\mathrm{Br} 1$ & $119.8(5)$ & $\mathrm{C} 11-\mathrm{C} 10-\mathrm{H} 10$ & 119.8 \\
\hline $\mathrm{C} 5-\mathrm{C} 4-\mathrm{C} 3$ & $120.9(6)$ & $\mathrm{C} 11-\mathrm{C} 10-\mathrm{C} 9$ & $120.4(7)$ \\
\hline $\mathrm{C} 3-\mathrm{C} 4-\mathrm{Br} 1$ & $119.3(5)$ & $\mathrm{C} 8-\mathrm{C} 9-\mathrm{H} 9$ & 120.3 \\
\hline $\mathrm{C} 2-\mathrm{C} 3-\mathrm{H} 3$ & 120.2 & $\mathrm{C} 10-\mathrm{C} 9-\mathrm{C} 8$ & $119.3(7)$ \\
\hline $\mathrm{C} 4-\mathrm{C} 3-\mathrm{C} 2$ & $119.5(6)$ & $\mathrm{C} 10-\mathrm{C} 9-\mathrm{H} 9$ & 120.3 \\
\hline
\end{tabular}




$\begin{array}{llll}\mathrm{C} 4-\mathrm{C} 3-\mathrm{H} 3 & 120.2 & \mathrm{C} 13-\mathrm{C} 12-\mathrm{H} 12 & 119.9 \\ \mathrm{C} 5-\mathrm{C} 6-\mathrm{C} 7 & 119.1(6) & \mathrm{C} 13-\mathrm{C} 12-\mathrm{C} 11 & 120.3(7) \\ \mathrm{C} 5-\mathrm{C} 6-\mathrm{C} 1 & 119.3(6) & \mathrm{C} 11-\mathrm{C} 12-\mathrm{H} 12 & 119.9 \\ \mathrm{C} 1-\mathrm{C} 6-\mathrm{C} 7 & 121.4(6) & \mathrm{C} 8-\mathrm{C} 13-\mathrm{H} 13 & 120.0 \\ \mathrm{C} 1-\mathrm{O} 1-\mathrm{H} 1 & 108(7) & \mathrm{C} 12-\mathrm{C} 13-\mathrm{C} 8 & 119.9(7) \\ \mathrm{C} 7-\mathrm{N} 1-\mathrm{C} 8 & 121.1(7) & \mathrm{C} 12-\mathrm{C} 13-\mathrm{H} 13 & 120.0 \\ \mathrm{C} 6-\mathrm{C} 7-\mathrm{H} 7 & 119.6 & \mathrm{C} 10-\mathrm{C} 11-\mathrm{C} 12 & 119.8(7) \\ \mathrm{N} 1-\mathrm{C} 7-\mathrm{C} 6 & 120.8(7) & \mathrm{C} 10-\mathrm{C} 11-\mathrm{H} 11 & 120.1 \\ \mathrm{~N} 1-\mathrm{C} 7-\mathrm{H} 7 & 119.6 & \mathrm{C} 12-\mathrm{C} 11-\mathrm{H} 11 & 120.1 \\ \mathrm{C} 2-\mathrm{C} 1-\mathrm{C} 6 & 120.2(7) & & \\ & & \mathrm{C} 7-\mathrm{C} 6-\mathrm{C} 1-\mathrm{C} 2 & 173.9(5) \\ \mathrm{Br} 1-\mathrm{C} 4-\mathrm{C} 3-\mathrm{C} 2 & -177.7(5) & \mathrm{C} 7-\mathrm{C} 6-\mathrm{C} 1-\mathrm{O} 1 & -5.2(9) \\ \mathrm{C} 5-\mathrm{C} 4-\mathrm{C} 3-\mathrm{C} 2 & -0.6(9) & \mathrm{C} 7-\mathrm{N} 1-\mathrm{C} 8-\mathrm{C} 9 & 38.4(11) \\ \mathrm{C} 5-\mathrm{C} 6-\mathrm{C} 7-\mathrm{N} 1 & -179.8(6) & \mathrm{C} 7-\mathrm{N} 1-\mathrm{C} 8-\mathrm{C} 13 & -146.6(7) \\ \mathrm{C} 5-\mathrm{C} 6-\mathrm{C} 1-\mathrm{C} 2 & -2.0(9) & \mathrm{C} 1-\mathrm{C} 2-\mathrm{C} 3-\mathrm{C} 4 & 0.0(9) \\ \mathrm{C} 5-\mathrm{C} 6-\mathrm{C} 1-\mathrm{O} 1 & 178.8(5) & \mathrm{C} 1-\mathrm{C} 6-\mathrm{C} 7-\mathrm{N} 1 & 4.2(10) \\ \mathrm{C} 4-\mathrm{C} 5-\mathrm{C} 6-\mathrm{C} 7 & -174.6(6) & \mathrm{C} 8-\mathrm{N} 1-\mathrm{C} 7-\mathrm{C} 6 & -171.0(6) \\ \mathrm{C} 4-\mathrm{C} 5-\mathrm{C} 6-\mathrm{C} 1 & 1.4(9) & \mathrm{C} 9-\mathrm{C} 10-\mathrm{C} 13-\mathrm{C} 11-\mathrm{C} 12 & -1.2(9) \\ \mathrm{C} 3-\mathrm{C} 2-\mathrm{C} 1-\mathrm{C} 6 & 1.3(9) & \mathrm{C} 13-\mathrm{C} 8-\mathrm{C} 9-\mathrm{C} 10 & -0.2(10) \\ \mathrm{C} 3-\mathrm{C} 2-\mathrm{C} 1-\mathrm{O} 1 & -179.5(5) & \mathrm{C} 13-\mathrm{C} 12-\mathrm{C} 11-\mathrm{C} 10 & 0.3(9) \\ \mathrm{C} 6-\mathrm{C} 5-\mathrm{C} 4-\mathrm{Br} 1 & 176.9(4) & \mathrm{C} 11-\mathrm{C} 10-\mathrm{C} 9-\mathrm{C} 8 & -0.7(10) \\ \mathrm{C} 6-\mathrm{C} 5-\mathrm{C} 4-\mathrm{C} 3 & -0.1(9) & \mathrm{C} 11-\mathrm{C} 12-\mathrm{C} 13-\mathrm{C} 8 & 0.4(9) \\ \mathrm{N} 1-\mathrm{C} 8-\mathrm{C} 9-\mathrm{C} 10 & 175.2(6) & -5(10) \\ \mathrm{N} 1-\mathrm{C} 8-\mathrm{C} 13-\mathrm{C} 12 & -176.3(6) & & \\ & & & \end{array}$

Hydrogen-bond geometry $\left(\AA,{ }^{\circ}\right)$

\begin{tabular}{lllll}
\hline$D-\mathrm{H} \cdots A$ & $D-\mathrm{H}$ & $\mathrm{H} \cdots A$ & $D \cdots A$ & $D-\mathrm{H} \cdots A$ \\
\hline $\mathrm{O} 1-\mathrm{H} 1 \cdots \mathrm{N} 1$ & $0.86(11)$ & $1.82(11)$ & $2.590(10)$ & $148(10)$ \\
\hline
\end{tabular}

